\title{
Decreased Drug Half Life
}

National Cancer Institute

\section{Source}

National Cancer Institute. Decreased Drug Half Life. NCI Thesaurus. Code C54607.

Decreased period of time required for the concentration or amount of drug in the body to be reduced to exactly one-half of a given concentration or amount. 